\title{
Air rifle breech injuries to digits: a preventable hazard
}

\author{
A. GRAY \\ Accident Unit, Countess of Chester Hospital, Chester, England
}

\section{SUMMARY}

The obvious injuries attributable to air rifles, namely those caused by pellets, are common and well documented (Batch, 1981; Reid, 1974; Rawson, 1965; Hutchinson, 1981). The author has encountered a series of injuries from air rifles involving a different mechanism. These commonly involve the digits, often in young people, and require considerable hospital time and resources in their treatment. They are essentially preventable.

\section{CASE REPORTS}

\section{Case 1}

A 22-year-old man sustained a deep laceration to the dorsum of his left index finger when the breech of a B.S.A. Meteor air rifle suddenly snapped shut over the tip of his finger when he accidentally pulled the trigger whilst loading the weapon. The laceration extended through the nail and nail bed and required removal of the nail and suturing of the nail bed under local anaesthetic (Fig. 1). The finger was dressed with bactigras and a tetanus toxoid booster was given. The wound healed after 2 weeks and full function was restored to the finger.

\section{Case 2}

A 10-year-old boy trapped the tip of his left thumb in the hinge at the base of the cocking lever of a B.S.A. Airsporter air rifle. The trigger had been pulled accidentally whilst cocking the weapon. The flap had been sutured at another hospital and an X-ray of the thumb had demonstrated an undisplaced fracture of the terminal phalanx of the thumb. The flap ultimately became avascular and required removal under a general

Correspondence: Mr Alastair Gray, Consultant in Accident and Emergency Medicine, Manchester Royal Infirmary, Manchester, England 


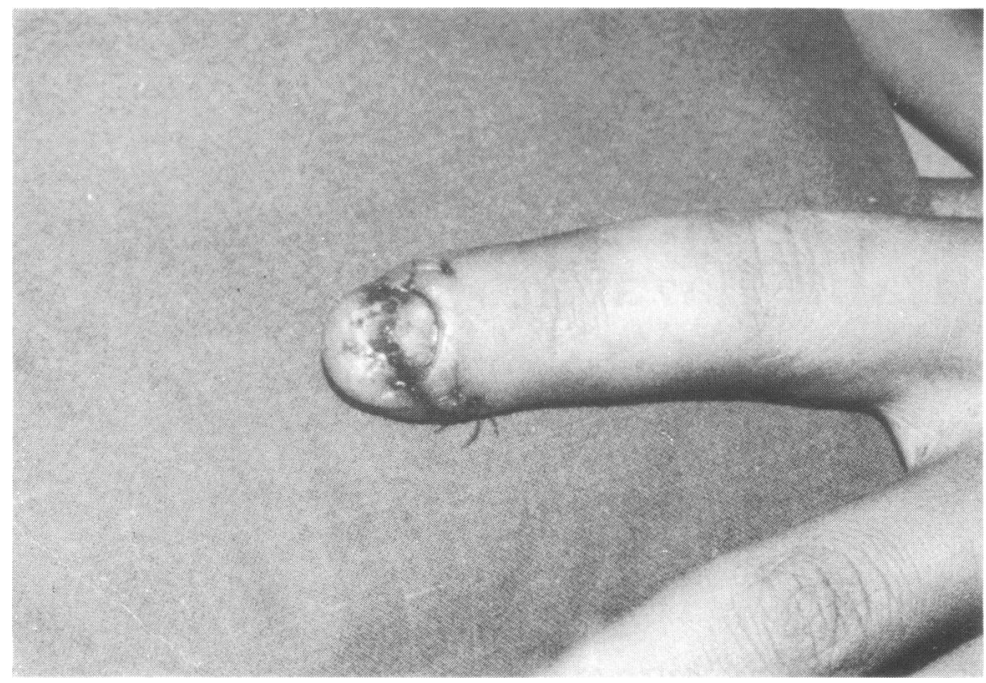

Fig. 1 Laceration of left index finger extending through nail bed following cleaning and suturing.

anaesthetic. The raw granulating area was covered with bactigras and ultimately heale $\frac{\mathbb{\infty}}{3}$ with full function 7 weeks following the injury.

\section{Case 3}

A 42-year-old man sustained a degloving injury to the tip of his right thumb when it was caught in the slide loading mechanism of a Weirauch air rifle. The wound was cleaned and dressed with jelonet and covered one week later with a split skin graft taken fron the ipsilateral forearm under a local anaesthetic. Five weeks following the injury the graft was fully healed and full function of the thumb had returned.

\section{DISCUSSION}

Air rifles require some form of cocking mechanism which can involve 'breaking' thể barrel and loading the pellet into the breech, or pulling down a lever under the barrel to tauten the spring and feeding the pellet through a sliding flap or rotating carrier into the breech. In all three mechanisms, if the trigger is pulled inadvertently when the air riffe is cocked but the breech is not closed, the breech mechanism will suddenly shut with some force. In all three of these cases a finger or thumb was caught in the breech mechanism when the trigger was inadvertently pulled. None of the air rifles mentione尔 was equipped with a safety mechanism to prevent the breech snapping shut in such fashion.

The instructions supplied with air rifles, which are often used by young an inexperienced people, sometimes with minimal supervision, do not draw sufficien $f$ 
attention to this potential hazard. The author suggests that all instructions should have clear warning notices regarding this problem, and that all air rifles should have some form of safety device to prevent the breech or cocking lever closing suddenly if the trigger is pulled. Finally, instructional courses should be available from organizations such as the British Association for Shooting and Conservation to the young in the use and safety of these weapons.

\section{ACKNOWLEDGEMENTS}

I would like to thank Mr J. Coals for permission to publish these cases.

\section{REFERENCES}

Batch J. G. (1981) The air rifle-a dangerous weapon. British Medical fournal 282, 1834.

Hutchinson G. H. (1981) Air gun injuries in children. Practitioner 225, 1058-60.

Rawson H. D. (1965) The air rifle-a potentially lethal weapon. New Zealand Medical fournal 64, 327.

Reid I. S. (1974) Airgun injuries in children. Medical fournal of Australia 1, 64-6. 